\title{
Neutrophil-to-lymphocyte and Platelet-to-lymphocyte Ratios in those with Pulmonary Embolism in the Course of Coronavirus Disease 2019
}

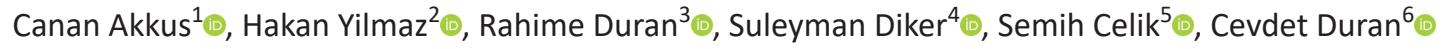

\begin{abstract}
Background: To investigate the levels of neutrophil-to-lymphocyte (NLR) and platelet-to-lymphocyte (PLR) ratios in those having a pulmonary embolism (PE) in the course of coronavirus disease 2019 (COVID-19).

Methods: The records of those having COVID-19 were retrospectively obtained from the hospital automation system. NLR and PLR were measured with the help of patients' blood cell counts.

Results: Of 1,452 COVID-19 patients, 17 (1.2\%) were diagnosed with PE. Compared with the controls, while leukocyte $(p=0.001)$, neutrophil $(p<0.001)$, and neutrophil percentages, $(p=0.001)$ and NLR $(p<0.001)$ and PLR $(p=0.006)$ had higher values, lymphocyte count $(p=0.004)$ and lymphocyte percentage $(p<0.001)$ showed lower values in the patients with PE.

Compared to the survivors, the non-survivors were found to have increased leukocyte $(p<0.001)$, neutrophil ( $p<0.001)$, and neutrophil percentages $(p<0.001)$, NLR $(p<0.001)$ and PLR $(p<0.001)$, and decreased lymphocyte $(p<0.001)$ counts and percentage $(p<0.001)$, hemoglobin $(p=0.005)$, hematocrit $(p=0.012)$, and platelet counts $(p<0.001)$.

While NLR and PLR cutoffs were found as 4.338 and 187.83 in predicting PE, the cutoff values of NLR and PLR were, respectively, 4.301 and 172.5 in predicting mortality.

The logistic regression analysis also revealed that all hematological parameters had no effects on the development of PE.

Conclusion: Although NLR and PLR had higher scores in PE patients, no relationship was determined between the levels of NLR and PLR and PE development. Further prospective studies including larger populations are required to enlighten the increased NLR and PLR in PE patients having COVID-19.

Keywords: COVID-19, Hematological changes, Neutrophil-to-lymphocyte ratio, Platelet-to-lymphocyte ratio, Pulmonary embolism. Indian Journal of Critical Care Medicine (2021): 10.5005/jp-journals-10071-23998
\end{abstract}

\section{HIGHLIGHTS}

- Increased NLR and PLR ratios are poor prognostic markers in both COVID-19 and PE.

- NLR and PLR displayed higher values in the COVID-19 cases with the mortal course who were detected to have PE.

- While the cutoff values of NLR and PLR were found as 4.338 and 187.83 in predicting PE, respectively, the cutoff values of NLR and PLR were 4.301 and 172.5 in predicting mortality, respectively.

- Further prospective studies including a large sample size are needed to elucidate the increased NLR and PLR in COVID-19 patients with PE.

\section{InTRODUCTION}

Along with coagulation, higher inflammation is a well-known characteristic of coronavirus disease 2019 (COVID-19). ${ }^{1,2}$ In many studies, arterial and venous thromboembolism risks have been revealed to be increased, and it can be speculated that increased inflammation and procoagulatory activity, decreased fibrinolysis, immobilization, and further unknown mechanisms may contribute to the increased risk. ${ }^{3}$

The neutrophil-to-lymphocyte (NLR) and platelet-to-lymphocyte (PLR) ratios are new and beneficial assets in the assessment of inflammation, disease activity, and severity in many clinical conditions. In the literature, several changes of hematological parameters, including leukocytosis, neutrophilia, lymphocytopenia,

\footnotetext{
${ }^{1,4-6}$ Department of Internal Medicine, Usak University School of Medicine, Usak, Turkey

${ }^{2}$ Department of Radiology, Usak University School of Medicine, Usak, Turkey

${ }^{3}$ Department of Internal Medicine, Tokat State Hospital, Tokat, Turkey

Corresponding Author: Cevdet Duran, Department of Internal Medicine, Usak University School of Medicine, Usak, Turkey, Phone: +02762212121, e-mail: drcduran@gmail.com

How to cite this article: Akkus C, Yilmaz H, Duran R, Diker S, Celik S, Duran C. Neutrophil-to-lymphocyte and Platelet-to-lymphocyte Ratios in those with Pulmonary Embolism in the Course of Coronavirus Disease 2019. Indian J Crit Care Med 2021;25(10):1133-1136.

Source of support: Nil

Conflict of interest: None
}

eosinophilia and thrombocytopenia, and the low levels of hemoglobin and hematocrit, and increased levels of NLR and PLR have been reported to be associated with the disease severity and poor prognosis in the patients with COVID-19. ${ }^{4-6}$ In the patients with a higher risk of acute pulmonary embolism (PE), NLR and PLR have also been demonstrated to be higher and related to mortality. ${ }^{7}$ As consistent with the findings of a meta-analysis performed by Telo et al., elevated NLR and PLR have been demonstrated to be strongly related to overall, short- and long-term mortality rates. ${ }^{8}$ 
In the literature, there is no study evaluating NLR and PLR so far in those developing PE in the course of COVID-19. Therefore, we here aimed at investigating hematological parameters of NLR and PLR in those developing PE in the course of COVID-19.

\section{Materials and Methods}

We conducted the current study in The Training and Research Hospital of Usak University between March 20 and December 11, 2020. Within the study having a retrospective design, the subjects were determined with the help of a positive severe acute respiratory syndrome coronavirus disease 2 (SARS-CoV-2) reverse transcriptase-polymerase chain reaction (RT-PCR) test, and our analysis was composed of those followed up because of COVID-19. The study was also approved by the local ethical board of the institution. The patients with any known solid organ involvements or hematological malignancies under 18 years of age and those receiving chemotherapy and immunosuppressive drugs were not included in the study. Of 1,479 patients diagnosed with SARS-CoV-2 positivity using the RT-PCR test between March 20 and December 11, 2020, 1,452 were included in the analysis. Patients' age, gender, hematological parameters, and disease outcomes were detected from the hospital's data and recorded on the patients' files. In the study, the rate of NLR was measured with the division of neutrophil counts to the lymphocyte counts, and PLR was also measured by dividing the platelet count to the lymphocyte counts. The BC-6800 automated-hematology analyzer (Mindray, Shenzhen, China) was used to measure hemogram, and PE was diagnosed with pulmonary angiography (Siemens, SOMATOM Definition AS 128-slice scanner, Munich, Germany).

\section{Statistical Analysis}

The analyses for normality were carried out with the ShapiroWilk test. While the Student's $t$-test was used to compare the continuous variables showing normal distribution in groups, the Mann-Whitney $U$ test was utilized to measure the non-normally distributed variables; however, the normally distributed continuous variables were presented as mean \pm standard deviation. Even so, the continuous variables without normal distribution were demonstrated as median (min:max). The analysis of the receiver operating characteristic (ROC) curve was performed to calculate the best cutoff values of NLR and PLR for detecting the development of PE and mortality. The binary regression analyses were also utilized to model the categoricaldependent variables. A $p$-value of 0.05 or less was accepted as significant.

\section{Results}

PE was diagnosed in 17 (1.2\%) of 1,452 COVID-19 patients. Compared to $\mathrm{PE}$ cases with the controls, it was seen that the mean age was higher [66 vs 58 , respectively $(p=0.036)]$, and male gender was prominent [13 (76.5\%) vs $676(47.1 \%)(p=0.016)]$ (Table 1). However, similar mortality rates were witnessed in the two groups $(p=0.107)$ (Table 1).

When compared to the controls, although the leukocyte $(p=0.001)$ and neutrophil counts $(p<0.001)$ and percentage $(p<0.001)$, and NLR $(p<0.001)$ and PLR $(p=0.006)$ showed higher values, the lymphocyte $(p=0.004)$ count and percentage $(p<0.001)$ were determined to be decreased in the patients with PE (Table 1). However, similar hemoglobin, hematocrit, and platelet counts were found in both groups.

Upon comparing to the survivors, non-survivors were seen to have increased leukocyte $(p<0.001)$, neutrophil $(p<0.001)$, neutrophil percentage $(p<0.001), \operatorname{NLR}(p<0.001)$ and PLR $(p<0.001)$ values, and decreased lymphocyte $(p<0.001)$ counts and percentage $(p<0.001)$, hemoglobin $(p=0.005)$, hematocrit $(p=0.012)$, and platelet counts $(p<0.001)$ (Table 2$)$.

As seen in the logistic regression analysis, all the abovementioned hematological parameters had no effects on the development of PE. In the ROC analysis, the levels of NLR were detected to be higher than 4.338 in predicting the PE development (sensitivity: $82.4 \%$ and specificity: 76.2\%) [area under the curve (AUC), 0.792; 95\% confidence interval $(\mathrm{Cl})$ : 0.699-0.884; $(p<0.001)$ ]. PLR levels were

Table 1: Baseline clinical and laboratory characteristics of COVID-19 patients with and without PE

\begin{tabular}{|c|c|c|c|}
\hline & Patients with PE $(n=17)$ & Controls $(n=1,435)$ & $p$ value \\
\hline Male/female $(n / n)(\% / \%)$ & $13 / 4(76.5 / 23.5)$ & $676 / 759(47.1 / 52.9)$ & 0.016 \\
\hline Age (year) & $66(21: 84)$ & $58(18: 94)$ & 0.036 \\
\hline BMI $\left(\mathrm{kg} / \mathrm{m}^{2}\right)$ & $27.54(22.49: 35.80)$ & $27.56 \pm 4.37$ & 0.878 \\
\hline Hospitalization time (day) & $17(4: 43)$ & $8.71 \pm 5.50$ & $<0.001$ \\
\hline Leukocyte count/ $\mu \mathrm{L}$ & $6,710(3,990: 13,850)$ & $5,814 \pm 2,772$ & 0.001 \\
\hline Neutrophil count/ $\mu \mathrm{L}$ & $5,290(2,270: 12,400)$ & $3,410(710: 43,140)$ & $<0.001$ \\
\hline Neutrophil percentage (\%) & $78.3(56.9: 94.9)$ & $66.1 \pm 12.5$ & $<0.001$ \\
\hline Lymphocyte count/ $\mu \mathrm{L}$ & $980(370: 2,560)$ & $1,370 \pm 977$ & 0.004 \\
\hline Lymphocyte percentage (\%) & $14.8(3.1: 30.1)$ & $25.6 \pm 11.2$ & $<0.001$ \\
\hline NLR & $5.14(1.89: 31.05)$ & $2.65(0.16: 52.03)$ & $<0.001$ \\
\hline PLR & $202.04(64.45: 1397.30)$ & $155.11(8.66: 3123.08)$ & 0.006 \\
\hline Hemoglobin (g/dL) & $13.6(10.0: 17.1)$ & $13.5 \pm 1.7$ & 0.838 \\
\hline Hematocrit (\%) & $40.4(31.5: 50.4)$ & $41.1 \pm 4.6$ & 0.915 \\
\hline MCV (80-100 fL) & $89.2(78.8: 95.6)$ & $86.5 \pm 6.6$ & 0.301 \\
\hline Platelet count $\times 10^{3} / \mu \mathrm{L}$ & $195(119: 517)$ & $200(13: 620)$ & 0.823 \\
\hline Outcomes (exitus letalis) [(n/total $n) / \%]$ & $3 / 17(17.6 \%)$ & $105 / 1,435(7.3 \%)$ & 0.107 \\
\hline
\end{tabular}

BMI, body mass index; COVID-19, coronavirus disease 2019; MCV, mean corpuscular volume; NLR, neutrophil-tolymphocyte ratio; PE, pulmonary embolism; PLR, platelet-to-lymphocyte ratio 
also found higher than 187.83 in predicting the PE development (sensitivity: $76.5 \%$ and specificity: $66.6 \%$ ) (AUC, $0.695 ; 95 \% \mathrm{Cl}$ : $0.568-0.822 ; p=0.006$ ). Also, while the levels of NLR were higher than 4.301 in predicting mortality (sensitivity: 63\% and specificity: 78.3\%) (AUC, $0.758 ; 95 \% \mathrm{Cl}: 0.709-0.808 ; p<0.001$ ), the PLR levels were detected to be higher than 172.50 in predicting the PE development (sensitivity: 63\% and specificity: 61.4\%) (AUC, 0.605; 95\% Cl: 0.540-0.670; $p<0.001)$.

\section{Discussion}

In the present study, although having no effects on the development of PE, it was detected that NLR and PLR values were higher among those with PE developing in the course of COVID-19 and among the non-survivors.

It was also demonstrated that elevated NLR and PLR values were superior to leukocytosis as an indicator of inflammation, disease severity, and prognosis in the course of COVID-19, as in many diseases. ${ }^{4-6}$ In a meta-analysis, including 20 studies performed on 3,508 patients, it was revealed that NLR $(p<0.01)$ and PLR $(p<0.001)$ levels were higher in those having the severe disease than the patients with the non-severe disease. ${ }^{6}$ Another retrospective study also emphasized that NLR is likely to be a significant prognostic biomarker of the results in critically ill COVID-19 patients. ${ }^{6}$ However, we found higher NLR and PLR values in the patients dying than the survivors in our study (Table 2). Also, compared to the survivors, the non-survivors were observed to have higher leukocyte, neutrophil, and neutrophil percentages, and lower lymphocyte counts and percentages, hemoglobin, hematocrit, and platelet counts. Since only three patients in the PE group died, no subgroup analysis was performed in terms of the presence of PE among the surviving and non-surviving patient groups.

Besides the outcomes reported by the abovementioned studies, many other studies have also revealed that the levels of NLR and PLR play a key role in showing disease severity and survival in acute PE patients. In a meta-analysis evaluating seven studies consisting of 2,323 patients, where the prognosis of NLR and PLR was assessed by Wang et al., increased NLR levels were revealed to be significantly associated with a nearly nine-fold increase for short-term mortality and a nearly ten-fold increased risk for overall (short- and long-term) mortality. Wang et al. also stated that the elevated levels of PLR were significantly associated with approximately six-fold increased overall and long-term mortality and seven-fold increased short-term mortality. ${ }^{8}$ In the study composed of 82 acute PE patients, PLR and NLR were found to be increased among those at high risk, and the PLR value may be useful in predicting the 3-month mortality, while NLR may be a beneficial marker in predicting the hospital mortality, 3-month mortality, and total 3-month mortality in those with acute PE. ${ }^{7}$ In another study carried out on 312 patients with the diagnosis of acute PE, NLR was shown as an available predictor for short-term mortality. ${ }^{9}$ Another study where Karatas et al. investigated PLR and NLR, the values of PLR (nearly 215 vs 139, $p<0.01$ ) and NLR (nearly 9 vs $4.2, p<0.01$ ) were reported to be further in non-survived patients with acute PE than those in the survivors. ${ }^{10}$ In the study by Soylu et al., the frequency rates of massive PE ( 66.2 vs $36.6 \%$, $p<0.001)$ and in-hospital mortality $(21.1 \%, 1.4 \%, p<0.001)$ were revealed to be elevated among those having high NLR. ${ }^{11}$ The same study also reported that the NLR value was found above 5.7 and related to 10.8 times higher mortality rate, compared to the NLR level under 5.7. Another study performed by Kayrak et al. reported that NLR values were higher in the non-survivor patients with acute $P E$ than the survivors $(16.5 \pm 14.6$ vs $6.6 \pm 6.9, p<0.001)$ and found NLR as 9.2 in predicting mortality. ${ }^{12}$ Although the NLR and PLR levels were detected to be further among PE patients within the study by Kayrak et al., we could demonstrate the effects of NLR and PLR levels on neither the development of PE nor mortality in our study. The existence of limited number of PE patients may have caused the effects of NLR and PLR not to be demonstrated in our study. In predicting mortality, on the contrary, while the NLR levels were found higher than 4.301 (sensitivity: 63\% and specificity: 78.3\%) (AUC, 0.758; 95\% Cl: 0.709-0.808; $p<0.001$ ), the PLR levels were detected to be higher than 172.50 (sensitivity: $63 \%$ and specificity: 61.4\%) (AUC, $0.605 ; 95 \% \mathrm{Cl}$ : 0.540-0.670; $p<0.001$ ) in our study.

Based on the literature and to our knowledge, we could encounter no article investigating the association of the NLR-PLR values with the development of PE among those with COVID-19. Therefore, our study aimed at determining the importance of NLR and PLR as prognostic markers among COVID-19 patients with PE. When COVID-19 patients with PE were compared to the controls in our study, although leukocyte, neutrophil, neutrophil percentage values, and NLR and PLR were found to be higher, lymphocyte counts and lymphocyte percentage were lower in the patients with PE. In the ROC analysis, while the NLR levels were detected to be higher

Table 2: Laboratory characteristics of survived and non-survived cases with COVID-19

\begin{tabular}{lccc}
\hline & Survived $(n=1,344)$ & Non-survived $(n=108)$ & $p$ value \\
\hline Leukocyte $/ \mu^{3} \mathrm{~L}$ & $5,220(1,880: 46,540)$ & $7,070(1,230: 35,710)$ & $<0.001$ \\
Neutrophil/ $\mu^{3} \mathrm{~L}$ & $3,375(710: 43,140)$ & $5,350(710: 24,630)$ & $<0.001$ \\
Neutrophil percentage (\%) & $65.9(15.4: 95.6)$ & $79.2(13.5: 96.9)$ & $<0.001$ \\
Lymphocyte/ $\mu^{3} \mathrm{~L}$ & $1,285(130: 4,300)$ & $895(260: 30,380)$ & $<0.001$ \\
Lymphocyte percentage (\%) & $25.7(2.2: 72.2)$ & $14.2(1.8: 85.1)$ & $<0.001$ \\
Hgb (g/dL) & $13.7(6.3: 18.8)$ & $13.2(5.3: 17.7)$ & 0.005 \\
Hematocrit $(\%)$ & $41.5(20.1: 55.1)$ & $40.3(19.7: 52.7)$ & 0.012 \\
Platelet $\times 10^{3} / \mu^{3} \mathrm{~L}$ & $200(20.9: 662)$ & $177.5(13: 608)$ & $<0.001$ \\
NLR & $2.54(0.33: 44.06)$ & $5.39(0.16: 52.03)$ & $<0.001$ \\
PLR & $153.71(25.18: 3123.08)$ & $192.04(8.66: 1,100)$ & $<0.001$ \\
\hline
\end{tabular}

COVID-19, coronavirus disease 2019; Hgb, hemoglobin; NLR, neutrophil-to-lymphocyte ratio; PLR, platelet-to-lymphocyte ratio 
than 4.338 in predicting the development of PE (sensitivity: $82.4 \%$ and specificity: 76.2\%) (AUC, 0.792; 95\%Cl: 0.699-0.884; $p<0.001$ ), the PLR levels were seen to be higher than 187.83 in predicting the development of PE (sensitivity: $76.5 \%$ and specificity: 66.6\%) (AUC, $0.695 ; 95 \% \mathrm{Cl}: 0.568-0.822 ; p=0.006)$. Even so, the logistic regression analysis revealed that all hematological parameters mentioned above had no effects on the development of PE.

\section{Conclusion}

Although the levels of NLR and PLR were demonstrated to be higher among PE patients, no relationship was demonstrated between the levels of NLR and PLR, and the PE development in our study. Based on the literature, there is no study evaluating NLR and PLR levels in those with PE developing in the course of COVID-19. We consider that our findings will be beneficial to prevent PE in those with COVID-19 or to diagnose PE earlier before its development; so, our study may contribute to the literature, and the NLR and PLR levels could also be benefited as preventive prognostic markers for COVID-19 patients. New and prospective studies with large populations are required to elucidate higher NLR and PLR in PE patients with COVID-19.

\section{Contributions by Authors}

$C A, H Y, R D, S D, S C$, and $C D$. supervised the design of the study and participated in collecting data and editing the manuscript.

\section{Acknowledgment}

The authors also thank Numan Duran for editing the manuscript.

\section{OrCID}

Canan Akkus @ https://orcid.org/0000-0003-4990-4927

Hakan Yilmaz @ib https://orcid.org/0000-0002-4710-7927

Rahime Duran (1) https://orcid.org/0000-0002-4100-8485

Suleyman Diker (1) https://orcid.org/0000-0002-1563-2743

Semih Celik (1) https://orcid.org/0000-0003-3786-1421

Cevdet Duran (1) https://orcid.org/0000-0001-9560-180X

\section{References}

1. Al-Ani F, Chehade S, Lazo-Langner A. Thrombosis risk associated with COVID-19 infection. A scoping review. Thromb Res 2020;192:152-160. DOI: 10.1016/j.thromres.2020.05.039.

2. Bompard F, Monnier H, Saab I, Tordjman M, Abdoul H, Fournier L, et al. Pulmonary embolism in patients with COVID-19 pneumonia. Eur Respir J 2020;56. DOI: 10.1183/13993003.01365-2020.

3. Liao SC, Shao SC, Chen YT, Chen YC, Hung MJ. Incidence and mortality of pulmonary embolism in COVID-19: a systematic review and metaanalysis. Crit Care 2020;24(1):464. DOI: 10.1186/s13054-020-03175-Z.

4. Kong M, Zhang H, Cao X, Mao X, Lu Z. Higher level of neutrophil-tolymphocyte is associated with severe COVID-19. Epidemiol Infect 2020;148:e139. DOI: 10.1017/S0950268820001557.

5. Yan X, Li F, Wang X, Yan J, Zhu F, Tang S, et al. Neutrophil to lymphocyte ratio as prognostic and predictive factor in patients with coronavirus disease 2019: a retrospective cross-sectional study. J Med Virol 2020;92(11):2573-2581. DOI: 10.1002/jmv.26061.

6. Chan AS, Rout A. Use of neutrophil-to-lymphocyte and platelet-tolymphocyte ratios in COVID-19. J Clin Med Res 2020;12(7):448-453. DOI: 10.14740/jocmr4240.

7. Telo S, Kuluozturk M, Deveci F, Kirkil G. The relationship between platelet-to-lymphocyte ratio and pulmonary embolism severity in acute pulmonary embolism. Int Angiol 2019;38(1):4-9. DOI: 10.23736/ S0392-9590.18.04028-2.

8. Wang Q, Ma J, Jiang Z, Ming L. Prognostic value of neutrophilto-lymphocyte ratio and platelet-to-lymphocyte ratio in acute pulmonary embolism: a systematic review and meta-analysis. Int Angiol 2018;37(1):4-11. DOI: 10.23736/S0392-9590.17.03848-2.

9. Ma Y, Mao Y, He X, Sun Y, Huang S, Qiu J. The values of neutrophil to lymphocyte ratio and platelet to lymphocyte ratio in predicting 30 day mortality in patients with acute pulmonary embolism. BMC Cardiovasc Disord 2016;16:123. DOI: 10.1186/s12872-016-0304-5.

10. Karatas MB, Ipek G, Onuk T, Güngör B, Durmuş G, Çanga $Y$, et al. Assessment of prognostic value of neutrophil to lymphocyte ratio and platelet to lymphocyte ratio in patients with pulmonary embolism. Acta Cardiol Sin 2016;32(3):313-320. DOI: 10.6515/ acs20151013a.

11. Soylu K, Gedikli O, Eksi A, Avcıoğlu Y, Soylu Al, Yüksel S, et al. Neutrophil-to-lymphocyte ratio for the assessment of hospital mortality in patients with acute pulmonary embolism. Arch Med Sci 2016;12(1):95-100. DOI: 10.5114/aoms.2016.57585.

12. Kayrak M, Erdogan HI, Solak Y, Akilli H, Gül EE, Yildirim O, et al. Prognostic value of neutrophil to lymphocyte ratio in patients with acute pulmonary embolism: a restrospective study. Heart Lung Circ 2014;23(1):56-62. DOI: 10.1016/j.hlc.2013.06.004. 